AIAA-99-1881

Verification and Analysis of Formulation 4 of Langley for the Study of Noise From High Speed Surfaces

\author{
F. Farassat \\ AIAA Associate Fellow, Senior Research Scientist \\ NASA Langley Research Center, Hampton, Virginia \\ Mark Farris \\ Midwestern State University, Wichita Falls, Texas
}

\begin{abstract}
$\underline{\text { Abstract }}$
There are several approaches to the prediction of the noise from sources on high speed surfaces. Two of these are the Kirchhoff and the Ffowcs williams-Hawkings methods. It can be shown that both of these methods depend on the solution of the wave equation with mathematically similar inhomogeneous source terms. Two subsonic solutions known as Formulation 1 and 1A of Langley are simple and efficient for noise prediction. The supersonic solution known as Formulation 3 is very complicated and difficult to code. Because of the complexity of the result, the computation time is longer than the subsonic formulas. Furthermore, it is difficult to assess the accuracy of noise prediction. We have been searching for a new and simpler supersonic formulation without these shortcomings. In the last AIAA Aeroacoustics Conference in Toulouse, Farassat, Dunn and Brentner presented a paper in which such a result was presented and called Formulation 4 of Langley. In this paper we will present two analytic tests of the validity this Formulation: i) the noise from dipole distribution on the unit circle whose strength varies radially with the square of the distance from the center and ii) the noise from dipole distribution on the unit sphere whose strength varies with the cosine of the angle from the polar axis. We will discuss the question of singularities of Formulation 4.
\end{abstract}

\section{Introduction}

Two common methods of noise prediction from moving surfaces are based on the Ffowcs WilliamsHawkings (FW-H) equation ${ }^{1}$ and the Kirchhoff formula for moving surfaces ${ }^{2}$. It can be shown that both these methods are based on the solution of wave equation with mathematically similar inhomogeneous source terms. The subsonic solutions known as Formulations 1 and 1A of Langley ${ }^{3,4,5}$ are simple and efficient to use on a computer. The supersonic result known as Formulation 3 is very complicated and difficult to code for noise prediction $^{6}$. Because of the complexity of this result, the execution time on a computer is long compared to the subsonic formulations. There are many surface geometric parameters, such as local normal curvature in various directions, in Formulation 3 which can not be physically interpreted. It is difficult to assess the accuracy of noise prediction because of the complexity of the computing algorithm. We have searched for simpler results for prediction of noise from sources on high speed surfaces.

Farassat, Dunn and Brentner have presented a new result in the last AIAA Aeroacoustics Conference in Toulouse which is considerably simpler than Formulation $3^{7}$. This result has been designated Formulation 4. The present paper continues the study of this new result. We apply Formulation 4 to two problems whose analytic solutions are known by other methods. These are: i) the noise from dipole distribution on the unit circle whose strength varies radially with the square of the distance from the center and ii) the noise from dipole distribution on the unit sphere whose strength varies with the cosine of the angle from the polar axis. We show that we do obtain the known analytic results and thus have validated Formulation 4.

We discuss the question of singularities of the new formulation which surprisingly is simpler to answer than those of Formulation 3. We was shown that the singularities are removable for $\mathrm{FW}-\mathrm{H}$ equation if we include the surface terms from the quadrupole source, and in the Kirchhoff formula for supersonic surfaces.

\section{The Governing Equation and Its Solution}

Given an open moving surface $f=0, \tilde{f}>0$, where $f=\tilde{f}=0$ denotes the edge of the panel, it can be shown that the governing differential equation for noise prediction by FW-H equation and the Kirchhoff method is 7 :

$$
\begin{aligned}
\square^{2} p^{\prime}= & q_{1} H(\tilde{f}) \delta(f)+{\underset{\sim}{q}}_{2} H(f) \delta_{s}^{\prime}(f) \\
& +q_{3} \delta(\tilde{f}) \delta(f)
\end{aligned}
$$


where the functions $q_{1}, q_{2}$ and $q_{3}$ are described in reference 7 . In this equation, $H($.$) is the Heaviside$ function, $\delta($.$) is the Dirac delta function and \delta^{\prime}{ }_{s}(f)$ is a distribution that picks up normal derivative of a test function on the surface $f=0$. The full solution of this equation (Formulation 4) is ${ }^{7}$ :

$$
\begin{aligned}
& 4 \pi p^{\prime}(\boldsymbol{x}, t) \\
& =\int_{\substack{F=0 \\
F>0}} \frac{1}{r}\left[\frac{q_{1}+\cot \theta \boldsymbol{t}_{1} \cdot \nabla_{2} q_{2}-\kappa_{1} q_{2}}{\Lambda}\right]_{r e t} d \Sigma \\
& +\int_{\begin{array}{c}
F=0 \\
\bar{F}
\end{array}=0} \frac{1}{r}\left[\frac{q_{3}+q_{2} \boldsymbol{v} \cdot \boldsymbol{t}_{1} \cot \theta}{\Lambda_{0}}\right]_{r e t} d L \\
& +\Sigma\left[\frac{4 q_{2}}{r \mid 1-M_{r}} \int_{0}^{\pi / 2} \frac{\operatorname{sig}[k(\varphi)]}{k_{r}-k(\varphi)} d \varphi\right]_{T}
\end{aligned}
$$

Here, $r=|\boldsymbol{x}-\boldsymbol{y}|,(\boldsymbol{x}, t)$ and $(\boldsymbol{y}, \tau)$ are the observer and the source space-time variables, respectively, and $\theta$ is the angle between the radiation direction $\hat{\boldsymbol{r}}=\boldsymbol{r} / \boldsymbol{r}$ and the local normal to $f=0$. The unit vector in the direction of projection of $\hat{\boldsymbol{r}}$ on the local tangent plane to the source surface is denoted $t_{1}$ and the local normal curvature of $f=0$ in the direction of $t_{1}$ is $\kappa_{1}$. the geodesic unit normal of the edge of the panel is $v$ and $\Lambda$ and $\Lambda_{0}$ are functions of the kinematic and geometric parameters of the panel ${ }^{7}$. We have defined $F=[f(\boldsymbol{y}, \tau)]_{\text {ret }}$ and $\tilde{F}=[\tilde{f}(\boldsymbol{y}, \tau)]_{\text {ret }}$. The last term only exists if the collapsing sphere $g=\tau-t-r / c=0$ leaves the panel tangentially at the point $T$. The signum function is denoted $\operatorname{sig}($.$) ,$ $k_{r}=1 / r$ and $k(\varphi)$ is the local normal curvature of the panel at $T$ as a function of azimuthal angle $\varphi$. The Mach number in the radiation direction is $M_{r}$. We mention here that Formulation 4 is valid at all Mach numbers although we intend to use it for surfaces moving at transonic and supersonic Mach numbers.

Note that we have issued a correction to the result presented in reference 7 . The correction appears in the electronic copy of this reference at NASA Langley Technical Report Server. The electronic address is given in the references below.

\section{$\underline{\text { Validation of Formulation } 4}$}

Since the part of the new formulation depending on $q_{1}$ and $q_{3}$ are simple and have been validated before ${ }^{6}$, we only need to validate the part depending on the source term $q_{2}$. We will again start with the differential equation and assume that the sources are stationary. It will be seen that these assumptions are necessary because we are seeking problems with analytic solutions and Formulation 4 is valid for both subsonic and supersonic surface sources. We consider two problems here.

\section{Example 1- Dipole Distribution on the Unit Circle}

We consider dipole distribution on the unit circle with the center at the origin of the $\mathrm{x}_{1} \mathrm{x}_{2}$-plane described by the following wave equation:

$$
\begin{gathered}
\square^{2} p^{\prime}=\frac{\partial}{\partial x_{3}}\left[q\left(x_{1}, x_{2}, t\right) \delta\left(x_{3}\right)\right] \\
=q\left(x_{1}, x_{2}, t\right) \delta^{\prime}\left(x_{3}\right) \\
q_{2}=-\left(1+\rho^{2}\right) e^{i \omega t} \\
\rho^{2}=x_{1}^{2}+x_{2}^{2}, \rho \leq 1
\end{gathered}
$$

The solution of this problem from classical mathematics is

$$
\begin{gathered}
4 \pi p^{\prime}(\boldsymbol{x}, t)= \\
x_{3} e^{i \omega t} \int_{0}^{1} \int_{0}^{2 \pi} \frac{e^{-i k r}}{r^{3}}\left(1+\rho^{2}\right)(1+i k r) \rho d \varphi d \rho
\end{gathered}
$$

where $(\rho, \varphi)$ is the polar coordinates in the $\mathrm{x}_{1} \mathrm{x}_{2}$-plane, $k=\omega / c$ and $r$ is the distance between the source and the observer. We will later integrate Eq. (4) numerically to compare with the results from Formulation 4.

Now we use Formulation 4, Eq. (2), for solving Eq. (3). Refer to Fig. 1 for definition of some symbols. Because of the symmetry of the problem with respect to the $x_{3}$-axis, we assume that the observer is in the $x_{1} x_{3}$ plane. We have the following relations:

$$
\begin{gathered}
r^{2}=\rho^{2}+x_{1}^{2}+x_{3}^{2}-2 \rho x_{1} \cos \varphi \\
r_{1}^{2}=\rho^{2}+x_{1}^{2}-2 \rho x_{1} \cos \varphi
\end{gathered}
$$

$\cos \theta=x_{3} / r, \sin \theta=r_{1} / r, \cot \theta=x_{3} / r_{1}(5-\mathrm{c}, \mathrm{d}, \mathrm{e})$

$$
\begin{gathered}
t_{1}=\left(\frac{x_{1}-\rho \cos \varphi}{r_{1}}, \frac{-\rho \sin \varphi}{r_{1}}, 0\right) \\
t_{1} \cdot \nabla q_{2}=-\frac{2 \rho e^{i \omega t}\left(x_{1} \cos \varphi-\rho\right)}{r_{1}}
\end{gathered}
$$




$$
\begin{gathered}
\kappa_{1}=0, \kappa_{g}=\left(1 / r_{1}\right), \\
v \cdot t_{1}=\left(1-x_{1} \cos \varphi\right) / r_{1}
\end{gathered}
$$

Using these results in Eq. (2), we get

$$
\begin{aligned}
p^{\prime}(\boldsymbol{x}, t)= & -\frac{x_{3} e^{i \omega t}}{2 \pi} \int_{0}^{1} \int_{0}^{2 \pi} \frac{\rho^{2} e^{-i k r}\left(x_{1} \cos \varphi-\rho\right)}{r r_{1}^{2}} d \varphi d \rho \\
& +\frac{x_{3} e^{i \omega t}}{2 \pi} \int_{0}^{2 \pi}\left[\frac{x_{1} \cos \varphi-1}{r_{1}^{2}} e^{-i k r}\right]_{\rho=1} d \varphi \\
& +\frac{e^{i \omega t}}{2}\left(1+x_{1}^{2}\right) e^{-i k x_{3}} H\left[1-\left|x_{1}^{2}\right|\right]
\end{aligned}
$$

The two expressions in Eq. (4) and Eq. (6) look very different from each other. We have used Mathematica 3 to compute $p^{\prime}(\boldsymbol{x}, t) e^{-i \omega t}$ from these two expressions for 11 values of $x_{1}$. In these calculations, shown in Table 1, we used $k=10, x_{2}=0$ and $x_{3}=5$. It is seen that the results from the two expressions are the same to a remarkable degree of accuracy.

This example validates Formulation 4 for a flat source surface. The next example applies this result to a curved surface.

\section{Example 2- Dipole Distribution on a Sphere}

We will consider a unit sphere $R=1$ with the center at the origin and a dipole distribution varying with the cosine of the angle $\Psi$ from the $\mathrm{x}_{3}$-axis. See Fig. 2 for some notation. We consider the following wave equation:

$$
\square^{2} p^{\prime}=-e^{i \omega t} \cos \Psi \delta^{\prime}(R-1)
$$

We use $(R, \Psi, \Phi)$ and $(\rho, \psi, \varphi)$ for the observer and the source variables, respectively. Let $r_{0}$ be the distance of the observer from the origin. Then, the solution of Eq. (7) in the geometric far field when the observer is on the positive $\mathrm{x}_{3}$-axis is:

$$
p^{\prime}(\boldsymbol{x}, t)=\frac{i \sin k}{r_{0}} e^{i\left(\omega t-k r_{0}\right)}
$$

Now, for the observer on the $\mathrm{x}_{3}$-axis and in the far field, Formulation 4, Eq. (6) gives:

$$
\begin{aligned}
p^{\prime}(x, t)= & \frac{1}{4 \pi r_{0}} \int\left[\cot \theta \boldsymbol{t}_{1} \cdot \nabla_{2} q_{2}\right]_{r e t} d S \\
& -\frac{1}{4 \pi r_{0}} \int\left[\left(\frac{\kappa_{1}}{\sin ^{2} \theta}+\kappa_{g} \cot \theta\right) q_{2}\right]_{r e t} d S \\
& -\left[\frac{\rho q_{2}}{2 r_{0}}\right]_{t-\left(r_{0}+1\right) / c}-\left[\frac{\rho q_{2}}{2 r_{0}}\right]_{t-\left(r_{0}-1\right) / c}
\end{aligned}
$$

Here, $q_{2}$ is the right side of Eq. (7) and $d S$ is element of the surface area of the sphere. We next use the following results in Eq. (9):

$$
\begin{gathered}
\theta=\psi, \kappa_{1}=-1, \rho=1, \kappa_{g}=\cot \psi \\
\boldsymbol{t}_{1} \cdot \nabla_{2} q_{2}=-\frac{\partial}{\partial \psi}-\left[\cos \psi e^{i \omega \tau}\right] \\
=-\sin \psi e^{i \omega \tau} \\
\frac{\kappa_{1}}{\sin ^{2} \theta}+\kappa_{g} \cot \theta=-1 \\
-\left[\frac{\rho q_{2}}{2 r_{0}}\right]_{t-\left(r_{0}+1\right) / c} \\
-\left[\frac{\rho q_{2}}{2 r_{0}}\right]_{t-\left(r_{0}-1\right) / c}=\frac{i \sin k}{r_{0}} e^{i\left(\omega t-k r_{0}\right)}
\end{gathered}
$$

In Eq. (11), the symbol $\tau$ stands for the source time that can be related to the angle $\psi$ on the surface of the sphere. When we use the above results in eq. (9), we get exactly the classical results Eq. (8). We have thus validated Formulation 4 for a curve surface also.

\section{Discussion of the Singularities}

One of the problems associated with supersonic surface sources is the appearance of singularities in the solution of wave equation. Some of these problems are purely mathematical in nature and their cause is the wrong choice of variables in the solution of the wave equation. There is also the possibility of physical singu- 
larities where no choice of variables can get rid of. We mention that both the thickness and loading sources on an open supersonic surface will have true singularities at some observer time. This problem was treated by Di Bernardis $^{8}$ and Farassat and Myers ${ }^{9}$. The latter authors showed that the inclusion of surface sources from the quadrupole source term of FW-H equation in the solution of this equation results in integrable singularities. We have shown that similar conclusion holds for the new formulation when applied to the solution of FW-H equation and the governing equation for the Kirchhoff formula for moving surfaces ${ }^{7}$.

The singularities of Formulation 3 for an open supersonic surface appear when part of its edge travels at supersonic speed in the plane normal to the edge. One can then construct the observer positions and the times that the singularity will be felt at the observer. The situation for Formulation 4 is somewhat different. First the singularities from the surface and line integrals are much simpler to analyze than those of Formulation 3 but of the same nature. Another cause of the appeance of singularities is due to the geometry of the source surface itself and is related to the formation of the caustic in geometric acoustics ${ }^{10}$. This type of singularity comes from the last term of Eq. (2). We will discuss the problem of singularities in a comprehensive paper on the new formulation later.

\section{$\underline{\text { Concluding Remarks }}$}

The purpose of this paper has been to validate Formulation 4 of Langley for prediction of noise from high speed moving surfaces. We have used two problems for which analytical solutions are available from classical analysis. We have shown that these solutions can be obtained also using the new formulation. The first problem is the radiation field of dipole distribution on a flat surface. We verified by a numerical study that the radiation field can be obtained by the new formulation. The second problem is radiation from dipole distribution on a sphere. To get an analytically simple expression from classical analysis, the observer is located in the far field and on $\mathrm{x}_{3}$-axis. We showed that this result could also be obtained by the new formulation.

The most significant fact about the new formulation is that it is much simpler than any previously known result in time domain for prediction of the noise from high speed surface sources. Furtheremore, because of the observer location, in the case of propfan noise calculations, none of the problems of singularities are present. This appears to be a major advance in noise prediction theory.

\section{Referensce}

1. Ffowcs Williams, J. E. and Hawkings, D.L., "Sound generation by turbulence and surfaces in arbitrary motion", Phil Trans. Roy. Soc. (London), 264A, 321-342 (1969)

2. Farassat, F., "The Kirchhoff formulas for moving surfaces in aeroacoustics-The subsonic and supersonic cases", NASA Technical Memorandum 110285 (1996), (Available at ftp:// techreports.larc.nasa.gov/pub/techreports/larc/96/ NASA-96-tm110285.ps.Z)

3. Farassat, F., "Theory of noise generation from moving bodies with an application to helicopter rotors", NASA TR R-451 (1975), (Available at http://techreports.larc.nasa.gov/ltrs/PDF/NASA-75-trr451.pdf)

4. Farassat F. and Succi, G. P., "The prediction of helicopter rotor discrete frequency noise", Vertica, 7, 309-320 (1983)

5. Brentner, Kenneth S., "Prediction of helicopter rotor discrete frequency noise-A computer program incorporating realistic blade motions and advanced acoustic formulation", NASA Technical Memorandum 87721 (1986)

6. Farassat, F., Padula, S. L. and Dunn, M. H., "Advanced turboprop noise prediction based on recent theoretical results", J. of Sound and Vib., 119 , 53-79 (1987)

7. Farassat, F., Brentner, Kenneth S. and Dunn, M. H., "A study of supersonic surface sources-The Ffowcs Williams-Hawkings equation and the Kirchhoff formula", AIAA Paper 98-2375 (1998) 
(Available at http://techreports.larc.nasa.gov/ltrs/ PDF/1998/aiaa/NASA-aiaa-98-237

8. Di Bernardis, Enrico: On a New Formulation for the Aeroacoustics of Rotating Blades (in Italian), Ph.D. Thesis, University of Romr (La Sapienza), 1989

9. Farassat, F. and Myers, M. K.: Line Source Singularity in the Wave equation and Its Removal by Quadrupole sources - a Supersonic Propeller Noise Problem, in Theoretical and Computational Acoustics, Volume 1, J. E. Ffowcs Williams, D. Lee, and A. D. Pierce (eds.), World Scientific Publishing, 1994

10. Pierce, Allan D.: Acoustics - An Introduction

to Its Physical Principles and Applications, Acoustical Society of America, 1989

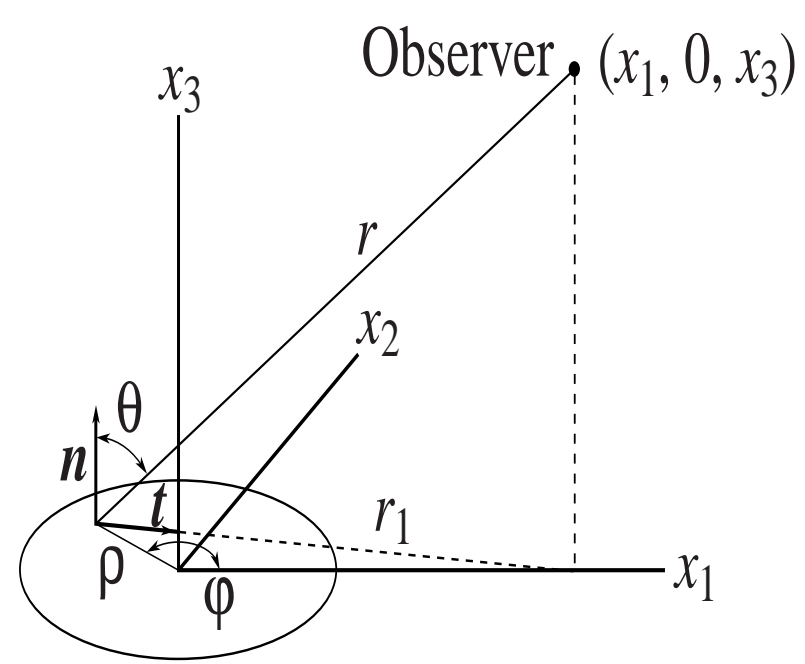

Figure 1. Definition of some symbols in Example 1

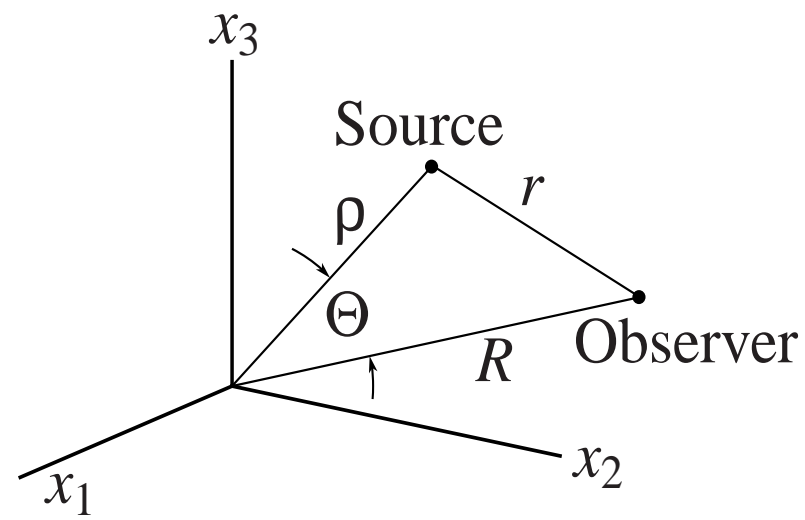

Figure 2. Definition of some symbols in Example 2 


\begin{tabular}{|c|c|c|}
\hline$x_{1}$ & Classical, Eq. (4) & $\begin{array}{l}\text { Formulation } 4 \\
\text { Eq. (6) }\end{array}$ \\
\hline 0 & $0.21098+0.673096 \mathrm{i}$ & $0.21098+0.673096 \mathrm{i}$ \\
\hline 0.25 & $0.23068+0.637725 \mathrm{i}$ & $0.238067+0.637724 \mathrm{i}$ \\
\hline 0.50 & $0.30169+0.531172 \mathrm{i}$ & $0.301689+0.531171 \mathrm{i}$ \\
\hline 0.75 & $0.355626+0.361800 \mathrm{i}$ & $0.355625+0.3618 \mathrm{i}$ \\
\hline 0.975 & $0.353059+0.184642 \mathrm{i}$ & $0.353058+0.184641 \mathrm{i}$ \\
\hline 0.995 & $0.34963+0.169323 \mathrm{i}$ & $0.349588+0.169312 \mathrm{i}$ \\
\hline 0.9995 & $0.348784+0.165908 \mathrm{i}$ & $0.348784+0.165908 \mathrm{i}$ \\
\hline 0.99995 & $0.348698+.165567 \mathrm{i}$ & $0.348698+0.165567 \mathrm{i}$ \\
\hline 1.00005 & $0.348679+0.165491 \mathrm{i}$ & $0.348679+0.165491 \mathrm{i}$ \\
\hline 1.25 & $0.263827+0.006876 \mathrm{i}$ & $0.263827+0.006876 \mathrm{i}$ \\
\hline 5 & $0.00397175-0.0118504 \mathrm{i}$ & $0.00397175+0.0118504 \mathrm{i}$ \\
\hline
\end{tabular}




\title{
VERIFICATION AND ANALYSIS OF FORMULATION 4 OF LANGLEY FOR THE STUDY OF NOISE FROM HIGH SPEED SURFACES
}

\section{F. Farassat}

NASA Langley Research Center, Hampton, Virginia Mark Farris

Midwestern State University, Wichita Falls, Texas

\section{Presented at}

The 5th AIAA/CEAS Aeroacoustics Conference Seattle, WA, 10-12 May 1999

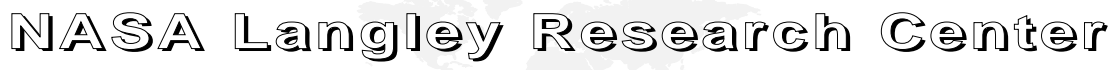




\section{Outline of the Talk}

- A short history of development of time domain formulations at Langley

- The governing wave equation

- Formulation 4

- Analytic validation by two examples

- The issue of singularities

- Concluding remarks 


\section{A Short History of Development of Time Domain Formulations at Langley}

We have been interested in helicopter rotor and propeller noise prediction since early 70's. We have developed ${ }^{*}$ :

i) Formulations 1 and $1 \mathrm{~A}$ for subsonic surface motion with Doppler factor- highly efficient for noise prediction

ii) Formulation 3 for subsonic, transonic and supersonic surface motion without the Doppler factor-very complicated, difficult to code and inefficient for noise prediction

We need a replacement for Formulation 3!

* Formulation 2 was abandoned because of its limitations 


\section{The Governing Wave Equation}

For sources on an open surface given by $f(x, t)=0, \tilde{f}(x, t)>0$, with edge defined by $f=\tilde{f}=0$, the governing equation for both FW-H and Kirchhoff methods is:

$$
\square^{2} p^{\prime}=q_{1} H(\tilde{f}) \delta(f)+{\underset{\sim}{2}}_{2} H(\tilde{f}) \delta^{\prime}(f)+q_{3} \delta(\tilde{f}) \delta(f)
$$

where $q_{1}, q_{2}$ and $q_{3}$ are functions of fluid mechanic and geometric parameters on the surface.

Mathematically, the most difficult source term is the one involving $q_{2}$. Formulation 4 is the solution of the above equation. 


\section{Formulation 4- The 4th AIAA/CEAS}

Aeroacoustics Conference in Toulouse, France 1998

$$
\begin{aligned}
4 \pi p^{\prime}(x, t) & =\int_{F=0} \frac{1}{r}\left[\frac{q_{1}+\cot \theta t_{1} \cdot \nabla_{2} q_{2}-\kappa_{1} q_{2}}{\Lambda}\right]_{r e t} d \Sigma \\
+\int_{\substack{F \\
\tilde{F}=0}} & \frac{1}{r}\left[\frac{q_{3}+q_{2} v \cdot t_{1} \cot \theta}{\Lambda_{0}}\right]_{r e t} d L+\Sigma\left[\frac{4 q_{2}}{r\left|1-M_{n}\right|} \int_{0}^{\pi / 2} \frac{\operatorname{sig}[k(\varphi)]}{k_{r}-k(\varphi)} d \varphi\right]_{T}
\end{aligned}
$$

Notation: $F=[f]_{r e t}, \tilde{F}=[\tilde{f}]_{r e t}, d \Sigma$ element of surface area of

$F=0, d L$ element of length of the edge $F=\tilde{F}=0, \hat{\boldsymbol{r}}=\boldsymbol{r} / \boldsymbol{r}$,

$\boldsymbol{r}=\boldsymbol{x}-\boldsymbol{y}, \boldsymbol{t}_{1}$ unit vector along projection of $\hat{\boldsymbol{r}}$ on the local tangent plane, $\theta$

angle between unit normal and radiation direction, $V$ local geodesic normal of the edge of panel, $k(\varphi)$ local normal curvature as a function of azimuthal angle $\varphi, M_{n}$ local normal Mach number, $\Lambda, \Lambda_{0}$ functions of geometric and kinematic parameters, $k_{r}=1 / r, \kappa_{1}$ local normal curvature along $\boldsymbol{t}_{1}, \mathbf{T}$ : tangency condition of collapsing sphere and the panel 


\section{Analytic Validation by Two Examples}

We will test Formulation 4 for the terms involving $q_{2}$ only. The other terms are simple and validated before (JSV,119,1987, 53-79). The two examples are:

i) Dipole distribution on unit circle whose strength varies with square of distance from center for arbitrary observer position, and

ii) Dipole distribution on unit sphere whose strength varies as cosine of the angle from $x_{3}$-axis for observer in the geometric far field and on the $x_{3}$-axis 


\section{Analytic Validation- Example 1}

Dipole distribution on unit

circle

$\square^{2} p^{\prime}=\frac{\partial}{\partial x_{3}}\left[q\left(x_{1}, x_{2}, t\right) \delta\left(x_{3}\right)\right]$

$$
=q\left(x_{1}, x_{2}, t\right) \delta^{\prime}\left(x_{3}\right)
$$

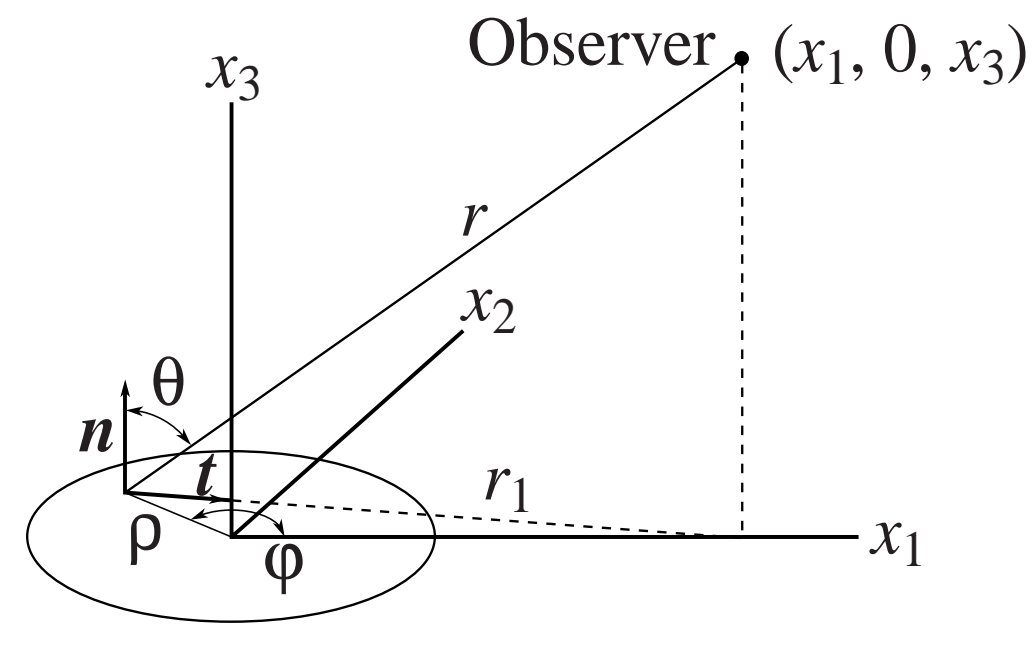

$q_{2}=-\left(1+\rho^{2}\right) e^{i \omega t}, \quad \rho^{2}=x_{1}^{2}+x_{2}^{2}, \quad \rho \leq 1, k=\omega / c$

Solution from classical mathematics

$$
4 \pi p^{\prime}(x, y)=x_{3} e^{i \omega t} \int_{0}^{1} \int_{0}^{2 \pi} \frac{e^{-i k r}}{r^{3}}\left(1+\rho^{2}\right)(1+i k r) \rho d \varphi d \rho
$$




\section{Analytic Validation- Example 1 (Cont'd)}

Solution from Formulation 4

$$
\begin{aligned}
p^{\prime}(\boldsymbol{x}, t)= & -\frac{x_{3} e^{i \omega t}}{2 \pi} \int_{0}^{1} \int_{0}^{2 \pi} \frac{\rho^{2} e^{-i k r}\left(x_{1} \cos \varphi-\rho\right)}{r r_{1}^{2}} d \varphi d \rho \\
& +\frac{x_{3} e^{i \omega t}}{2 \pi} \int_{0}^{2 \pi}\left[\frac{x_{1} \cos \varphi-1}{r_{1}^{2}} e^{-i k r}\right]_{\rho=1} d \varphi \\
& +\frac{e^{i \omega t}}{2}\left(1+x_{1}^{2}\right) e^{-i k x_{3}} H\left[1-\left|x_{1}^{2}\right|\right]
\end{aligned}
$$

$H$ ( ) Heaviside function 


\title{
Analytic Validation- Example 1 (Cont'd)
}

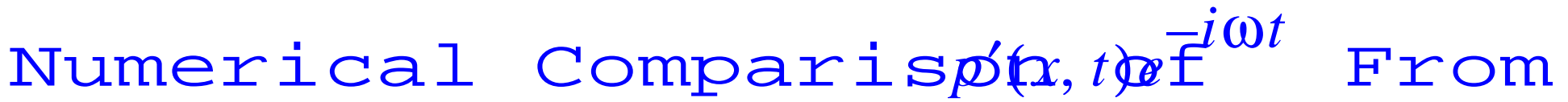 \\ Classical Method and Formulation$$
k=10, x_{2}=0, x_{3}=5
$$

\begin{tabular}{|c|c|c|}
\hline$x_{1}$ & Classical Solution & Formulation 4 \\
\hline $\mathbf{0}$ & $0.21098+0.673096 \mathrm{i}$ & $0.21098+0.673096 \mathrm{i}$ \\
\hline 0.25 & $0.23068+0.637725 \mathrm{i}$ & $0.238067+0.637724 i$ \\
\hline 0.50 & $0.30169+0.531172 i$ & $0.301689+0.531171 \mathrm{i}$ \\
\hline 0.75 & $0.355626+0.361800 i$ & $0.355625+0.361800 i$ \\
\hline 0.975 & $0.353059+0.184642 i$ & $0.353058+0.184641 i$ \\
\hline 0.995 & $0.34963+0.169323 \mathrm{i}$ & $0.349588+0.169312 i$ \\
\hline 0.9995 & $0.348784+0.165908 i$ & $0.348784+0.165908 i$ \\
\hline 0.99995 & $0.348698+.165567 i$ & $0.348698+0.165567 i$ \\
\hline 1.00005 & $0.348679+0.165491 i$ & $0.348679+0.165491 \mathrm{i}$ \\
\hline 1.25 & $0.263827+0.006876 i$ & $0.263827+0.006876 i$ \\
\hline 5 & $0.00397175+0.0118504 i$ & $0.00397175+0.0118504 i$ \\
\hline
\end{tabular} \\ The agreement of the two solutions is Excellent!
}




\section{Analytic Validation-Example 2}

Dipole distribution on

unit sphere, $\Psi$ angle from

$x_{3}$-axis

$\square^{2} p^{\prime}=-e^{i \omega t} \cos \Psi \delta^{\prime}(R-1)$

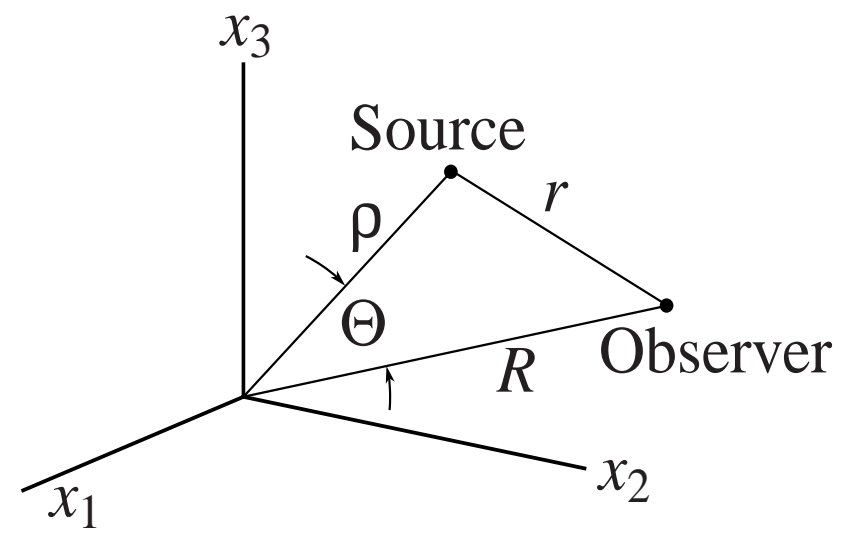

Solution from classical mathematics for observer in the geometric far field and on $\mathrm{x}_{3}$-axis, $r_{0}$ observer distance from origin

$$
p^{\prime}(\boldsymbol{x}, t)=\frac{i \sin k}{r_{0}} e^{i\left(\omega t-k r_{0}\right)}
$$




\section{Analytic Validation- Example 2 (Cont'd)}

Solution from formulation 4 (before far field approximation and locating the observer on the $\mathrm{x}_{3}$-axis)

$$
\begin{aligned}
p^{\prime}(x, t)= & \frac{1}{4 \pi r_{0}} \int\left[\cot \theta t_{1} \cdot \nabla_{2} q_{2}\right]_{r e t} d S \\
& -\frac{1}{4 \pi r_{0}} \int\left[\left(\frac{\kappa_{1}}{\sin ^{2} \theta}+\kappa_{g} \cot \theta\right) q_{2}\right]_{r e t} d S \\
& -\left[\frac{\rho q_{2}}{2 r_{0}}\right]_{t-\left(r_{0}+1\right) / c}-\left[\frac{\rho q_{2}}{2 r_{0}}\right]_{t-\left(r_{0}-1\right) / c}
\end{aligned}
$$

When the observer approximation is made, the two solutions are in agreement! 


\section{The Issue of Singularities}

- The singularities of Formulation 4 are of lower order than Formulation 3 and easier to analyze

- A new kind of singularity depending on the geometry of the surface and related to the formation of caustic in geometrical acoustics appear in Formulation 4

- The solution of FW-H Eq. when surface sources from quadrupole term are added to thickness and loading terms is singularity free for supersonic surfaces 


\section{Concluding Remarks}

- We have validated Formulation 4 using two examples whose analytic solutions are known from classical mathematics

- Formulation 4 is the simplest result we know for prediction of noise from sources on high speed surfaces

- The analysis of singularities of the new formulation is much simpler than those of Formulation 3

- Formulation 4 is useful in both the $\mathrm{FW}-\mathrm{H}$ and Kirchhoff methods 